\title{
Identification the Highest Risk of Performance Based Contract in Bojonegoro-Padangan Road Projects
}

\author{
Eko Prihartanto ${ }^{1}$, M. Djaya Bakri ${ }^{1}$ \\ Civil Engineering Departement, The Borneo Tarakan University ${ }^{I}$
}

\begin{abstract}
Development of road facilities and infrastructure in order to meet the need to improve the welfare of the community. Contract of project activity is carried out to bind both parties in this case, Owner and contractor as an interested party has the purpose to bind the rights and responsibilities in completing project activities. Performance based contracts are the innovative contracts presented by the Indonesian government to improve the quality of construction of road construction. The risk of applying this type of contract will be investigated so that it will be known to the highest risk, given the performance based contract has 4 stages such as design, build, operate, and maintenance. The project that implements the performance based contract under study is the Bojonegoro-Padangan road project, East Java. In the process of knowing the highest risk variable that occurs in the implementation, the researcher uses a simple probabilistic concept to determine the highest risk of each stage of a performance based contract. The correspondent of the contractor is PT Pembangunan Pembangunan (Persero) Tbk - PT Basuki Rahmanta Putra (Joint Operational) on the road construction project Bojonegoro - Padangan, East Java. The results obtained from this study from the implementation of performance-based contracts on the Bojonegoro-Padangan road construction project with the highest risk event gain is at the Design stage: Project budget. Build Stage: The vendor's offer price is higher than estimated. Operate Phase: Cashflow delay. Maintenance Stage: Short-term focus fails to minimize long-term costs..
\end{abstract}

Keywords-Construction, Contract, Maintenance, Road.

\section{INTRODUCTION}

The Indonesian government has implemented a performance based contract as a form of contract that is expected to provide innovation on the quality of construction. The adoption of this contract has not been fully implemented as performance-based contracts are still under probation, the risks of their application are not fully known. Performance based contracts have 4 stages, such as Design, Build, Operate, and Maintenance. All of these stages have different risks when applied. The implementation of the Bojonegoro-Padangan road construction that has implemented this contract becomes the object of the researcher to know the risks that occur at each stage. Projects that have entered this maintenance phase will also provide an overview of the dominant risks that occur during implementation. Unknown information about the risks that arise from the implementation of performance based contracts is an opportunity for research, and developed to determine the probability of events. Therefore, it can be used as a consideration for the government to be applied in various regions in Indonesia.

\section{I.1 Problem Formulation}

How is the highest risk of each stage of performance based contracts implemented in the BojonegoroPadangan road construction project be known?

\section{I.2 Aim}

Aims to perform the highest risk analysis of each stage of performance based contracts implemented in the Bojonegoro-Padangan road construction project.

\section{I.3 Limitations of research}

a. Risks that occur in Performance Based Contract for road projects.

b. Respondents in this research is the provider of goods / services that is the main contractor in the activities on the national road project using Performance Based Contract.

c. Details of the risks and studied were obtained from preliminary literature and surveys.

\section{LITERATURE STUDY}

II.1 Forms of Construction Contracts

The form of construction contract according to (1) can be reviewed from various aspects, namely:

a. Aspect Of Cost Calculation

1. Fixed Lump Sum Price

2. Unit Price

b. Aspect of Service Calculation

1. Cost Without Fee

2. Cost Plus Fee

3. Cost Plus Fixed Fee

c. Aspects of Payment Method

1. Monthly Payment

2. Stage Payment

3. Contractor's Full Prefinanced

d. Task Division Aspects

1. Conventional Contract

2. Specialist Contract

3. Design Construct / Build, Turnkey

4. Engineering, Procurement and Construction

5. Build, Operate and Transfer

6. Force Account

\section{2 Performance Based Contract}

Performance Based Contract (PBC) is a type of contract with a clear objective and indicator that bases payments on the fulfillment of minimum performance indicators. 
Critical elements of effective PBC are well defined and clearly defined job statements in order to achieve performance standards (2)

\section{II.3 Risk}

(3) explained in his book entitled Risk Management for contractors, which explained that Risk is a variation in things that may occur naturally in a situation. Meanwhile, according to (4) Risk is a threat to life, property or financial benefits due to the dangers that occur. In general, risk is associated with probability of occurrence beyond the expected events

II.4 Risk Relationship on Performance Based Contracts According to (5) explains To know the relationship of risk at the Performance Based Contract stage identified risk will be known first variables and factors to be analyzed at each stage.

\section{II.5 Road}

Government Regulation No. 34 Year 2006 describes the Road is a land transportation infrastructure covering all parts of the road, including auxiliary buildings and equipment intended for traffic, located on the surface of the soil, above ground level, below ground and / or water, and On the water surface, except for railroads, lorries, and cable roads.

\section{II.6 Simple Probabilistic Concepts}

According to (6) an approach is developed using two criteria that are important to measure risk, namely:

1. Probability is the possibility of an undesirable event.

2. Impact is the level of influence or size of the impact on other activities, if unexpected events occur.

According to (7) the value of risk is the result of multiplication of the probability value of risk with the value of risk impact.

Table. 1

Score of Impact

Risk assessment can be formulated as follows:

$\mathrm{R}=\mathrm{P} \times \mathrm{I}$

Where :

$\mathrm{R}=$ Risk level

$\mathrm{P}=$ potential risk

\begin{tabular}{ccccc}
\hline \multirow{2}{*}{ Description } & Probability & \multicolumn{2}{c}{ Impact on Project } & \\
\cline { 3 - 4 } & & $\begin{array}{c}\text { Timescale } \\
(\% \text { late })\end{array}$ & $\begin{array}{c}\text { Cost } \\
(\% \text { increase })\end{array}$ & Score \\
\hline VLO & $<10 \%$ & $<10 \%$ & $<5 \%$ & 1 \\
\hline LO & $10-30 \%$ & $10-20 \%$ & $5-10 \%$ & 2 \\
\hline MED & $30-50 \%$ & $20-40 \%$ & $10-15 \%$ & 3 \\
\hline HI & $50-70 \%$ & $40-50 \%$ & $15-30 \%$ & 4 \\
\hline VHI & $>70 \%$ & $>50 \%$ & $>30 \%$ & 5 \\
\hline
\end{tabular}

$\mathrm{I}=$ the level of risk impact

With this formula the risk assessment is not based on absolute estimation, but Use the interval class as shown in table 1 as follows:

Table. 2

Probability Impact Grid

\begin{tabular}{|c|c|c|c|c|c|c|}
\hline \multirow{4}{*}{$\begin{array}{c}\text { H } \\
\text { MED } \\
\text { L } \\
\text { VL }\end{array}$} & 4 & 4 & 8 & 12 & 16 & 20 \\
\hline & 3 & 3 & 6 & 9 & 12 & 15 \\
\hline & 2 & 2 & 4 & 6 & 8 & 10 \\
\hline & 1 & 1 & 2 & 3 & 4 & 5 \\
\hline & & 1 & 2 & 3 & 4 & 5 \\
\hline & & SL & $\mathbf{L}$ & MED & H & VH \\
\hline
\end{tabular}

Table. 3

Risk Level

\begin{tabular}{cc}
\hline Score & Risk \\
\hline $1-5$ & Very Low \\
\hline $5-10$ & Low \\
\hline $10-15$ & Medium \\
\hline $15-20$ & High \\
\hline $20-25$ & VeryHigh \\
\hline
\end{tabular}

Source (8)

\section{METHOD}

The concept of this descriptive study to perform the highest risk analysis at each stage of Performance Based Contract on the project that has been implemented.

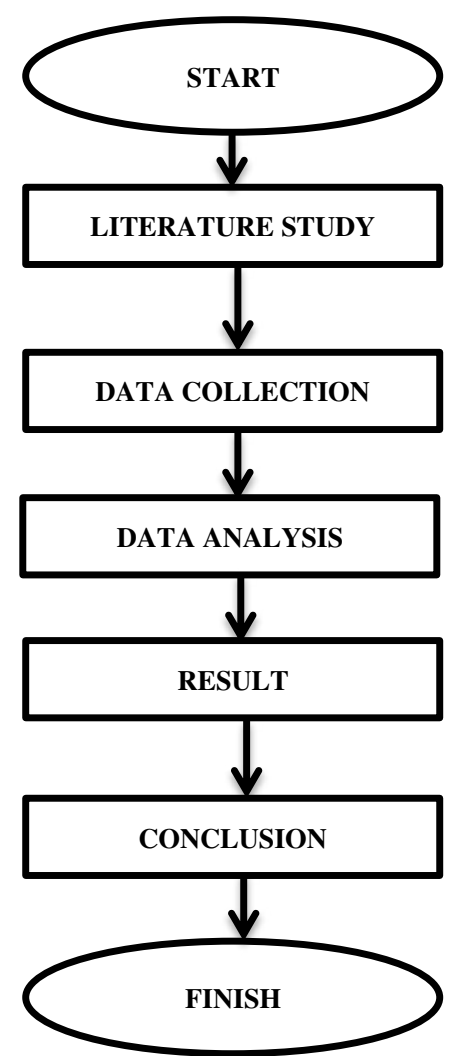

Figure.1 Flowchart of Research

\section{III.1 Data collection}

Data collection has been carried out from research references related to the subject and object of research, from the references such as journals, textbooks, theses, seminars and research reports.

Primary data collection has been done by researchers by:

1. Interview

2. Questionnaire

Data has been obtained after the construction phase during the maintenance service period with 1643 
calendar days from the year 2015 until 2019 on the Bojonegoro-Padangan road project.

\section{III.2 Respondent}

Respondent in this research is contractor who apply contract or Performance Based Contract in East Java area is PT Pembangunan Pembangunan (Persero) Tbk - PT Basuki Rahmanta Putra (Joint Operational).

\section{RESULTS AND DISCUSSION}

Assessment analysis for risk has been obtained from questionnaires that have been processed from risk variables arising from contractors who have implemented.

Table. 4 Risk Event Analysis

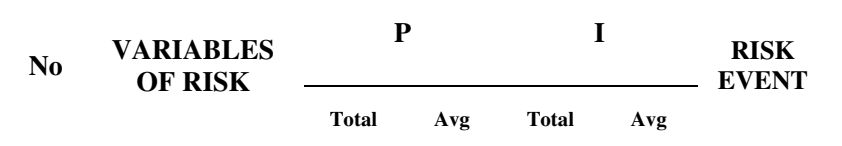

PT. PP (CONTRACTOR)

\begin{tabular}{|c|c|c|c|c|c|c|}
\hline $\mathbf{A}$ & DESIGN & & & & & \\
\hline 1 & $\begin{array}{l}\text { Accuracy scope } \\
\text { of work }\end{array}$ & 20 & 2.857 & 23 & 3.286 & 9.388 \\
\hline 2 & $\begin{array}{l}\text { Qualification } \\
\text { engineer }\end{array}$ & 21 & 3 & 18 & 2.571 & 7.714 \\
\hline 3 & $\begin{array}{l}\text { Engineering } \\
\text { communication } \\
\text { with } \\
\text { procurement } \\
\end{array}$ & 21 & 3 & 17 & 2.429 & 7.286 \\
\hline 4 & $\begin{array}{l}\text { Use of } \\
\text { technology for } \\
\text { working } \\
\text { methods } \\
\end{array}$ & 23 & 3.286 & 17 & 2.429 & 7.980 \\
\hline 5 & Project budget & 23 & 3.286 & 22 & 3.143 & 10.327 \\
\hline 6 & $\begin{array}{l}\text { Project } \\
\text { implementation } \\
\text { schedule }\end{array}$ & 19 & 2.714 & 19 & 2.714 & 7.367 \\
\hline 7 & Design changes & 15 & 2.143 & 17 & 2.429 & 5.204 \\
\hline 8 & $\begin{array}{l}\text { Incomplete } \\
\text { specs }\end{array}$ & 15 & 2.143 & 17 & 2.429 & 5.204 \\
\hline 9 & $\begin{array}{l}\text { Shop Drawing } \\
\text { incomplete }\end{array}$ & 16 & 2.286 & 17 & 2.429 & 5.551 \\
\hline 10 & $\begin{array}{l}\text { Lack of design } \\
\text { accuracy }\end{array}$ & 19 & 2.714 & 18 & 2.571 & 6.980 \\
\hline 11 & $\begin{array}{l}\text { Less } \\
\text { sophisticated } \\
\text { design and } \\
\text { engineering }\end{array}$ & 15 & 2.143 & 13 & 1.857 & 3.980 \\
\hline B & BUILD & & & & & \\
\hline 1 & $\begin{array}{l}\text { The vendor's } \\
\text { offer price is } \\
\text { higher than } \\
\text { estimated }\end{array}$ & 24 & 3.429 & 26 & 3.714 & 12.735 \\
\hline 2 & $\begin{array}{l}\text { Availability of } \\
\text { materials and } \\
\text { human } \\
\text { resources }\end{array}$ & 8 & 1.143 & 15 & 2.143 & 2.449 \\
\hline 3 & $\begin{array}{l}\text { Delays in the } \\
\text { supply of } \\
\text { materials and } \\
\text { tools }\end{array}$ & 15 & 2.143 & 11 & 1.571 & 3.367 \\
\hline 4 & $\begin{array}{l}\text { Identification of } \\
\text { materials and } \\
\text { equipment }\end{array}$ & 15 & 2.143 & 11 & 1.571 & 3.367 \\
\hline 5 & $\begin{array}{l}\text { Vendor Quality } \\
\text { Control }\end{array}$ & 9 & 1.286 & 8 & 1.143 & 1.469 \\
\hline
\end{tabular}

\begin{tabular}{|c|c|c|c|c|c|c|}
\hline 6 & $\begin{array}{l}\text { Procurement of } \\
\text { control } \\
\text { documents }\end{array}$ & 15 & 2.143 & 7 & 1 & 2.143 \\
\hline 7 & $\begin{array}{l}\text { Manufacturing } \\
\text { process }\end{array}$ & 15 & 2.143 & 12 & 1.714 & 3.673 \\
\hline 8 & $\begin{array}{l}\text { Vendor } \\
\text { Performance }\end{array}$ & 11 & 1.571 & 7 & 1 & 1.571 \\
\hline 9 & $\begin{array}{l}\text { Material } \\
\text { warranty }\end{array}$ & 12 & 1.714 & 13 & 1.857 & 3.184 \\
\hline 10 & $\begin{array}{l}\text { Late approval } \\
\text { from the owner }\end{array}$ & 14 & 2 & 11 & 1.571 & 3.143 \\
\hline 11 & $\begin{array}{l}\text { Disputes from } \\
\text { third parties }\end{array}$ & 11 & 1.571 & 11 & 1.571 & 2.469 \\
\hline 12 & $\begin{array}{l}\text { Less experience } \\
\text { in inspection } \\
\text { and shipping } \\
\end{array}$ & 14 & 2 & 7 & 1 & 2.000 \\
\hline $\mathbf{C}$ & OPERATE & & & & & \\
\hline 1 & $\begin{array}{l}\text { Site conditions } \\
\text { different from }\end{array}$ & 18 & 2.571 & 14 & 2 & 5.143 \\
\hline 2 & $\begin{array}{l}\text { Restricting } \\
\text { working hours }\end{array}$ & 14 & 2 & 11 & 1.571 & 3.143 \\
\hline 3 & $\begin{array}{l}\text { Quality control } \\
\text { and ansurance }\end{array}$ & 10 & 1.429 & 11 & 1.571 & 2.245 \\
\hline 4 & $\begin{array}{l}\text { The design can } \\
\text { not be applied } \\
\text { in the field }\end{array}$ & 15 & 2.143 & 11 & 1.571 & 3.367 \\
\hline 5 & $\begin{array}{l}\text { Additional time } \\
\text { due to rework }\end{array}$ & 13 & 1.857 & 15 & 2.143 & 3.980 \\
\hline 6 & Design changes & 14 & 2 & 19 & 2.714 & 5.429 \\
\hline 7 & $\begin{array}{l}\text { The supply of } \\
\text { materials from } \\
\text { third parties } \\
\text { does not meet } \\
\text { specifications }\end{array}$ & 12 & 1.714 & 14 & 2 & 3.429 \\
\hline 8 & Forced mature & 12 & 1.714 & 15 & 2.143 & 3.673 \\
\hline 9 & $\begin{array}{l}\text { Friendship } \\
\text { supervisor in } \\
\text { making } \\
\text { decisions } \\
\end{array}$ & 14 & 2 & 15 & 2.143 & 4.286 \\
\hline 10 & Cash flow delay & 19 & 2.714 & 19 & 2.714 & 7.367 \\
\hline 11 & $\begin{array}{l}\text { Disturbance } \\
\text { from the } \\
\text { surrounding } \\
\text { environment }\end{array}$ & 21 & 3 & 16 & 2.286 & 6.857 \\
\hline 12 & $\begin{array}{l}\text { Disputes } \\
\text { regarding the } \\
\text { definition of } \\
\text { specifications } \\
\text { and documents }\end{array}$ & 16 & 2.286 & 17 & 2.429 & 5.551 \\
\hline 13 & $\begin{array}{l}\text { Duration in } \\
\text { project } \\
\text { implementation }\end{array}$ & 12 & 1.714 & 15 & 2.143 & 3.673 \\
\hline 14 & $\begin{array}{l}\text { Differences in } \\
\text { availability of } \\
\text { budgets with } \\
\text { work progress }\end{array}$ & 19 & 2.714 & 18 & 2.571 & 6.980 \\
\hline 15 & $\begin{array}{l}\text { Quality of work } \\
\text { does not meet } \\
\text { the job }\end{array}$ & 15 & 2.143 & 15 & 2.143 & 4.592 \\
\hline 16 & $\begin{array}{l}\text { Unpredictable } \\
\text { soil conditions }\end{array}$ & 16 & 2.286 & 16 & 2.286 & 5.224 \\
\hline 17 & $\begin{array}{l}\text { Inadequate } \\
\text { specifications }\end{array}$ & 14 & 2 & 15 & 2.143 & 4.286 \\
\hline 18 & $\begin{array}{l}\text { Delayed } \\
\text { payment termin } \\
\text { progress }\end{array}$ & 19 & 2.714 & 17 & 2.429 & 6.592 \\
\hline 19 & $\begin{array}{l}\text { Licensing and } \\
\text { regulation }\end{array}$ & 16 & 2.286 & 17 & 2.429 & 5.551 \\
\hline 20 & $\begin{array}{l}\text { The } \\
\text { postponement is } \\
\text { entangled in } \\
\text { disputes }\end{array}$ & 14 & 2 & 14 & 2 & 4.000 \\
\hline 21 & $\begin{array}{l}\text { Differences in } \\
\text { terms of }\end{array}$ & 14 & 2 & 14 & 2 & 4.000 \\
\hline
\end{tabular}




\begin{tabular}{|c|c|c|c|c|c|c|}
\hline & $\begin{array}{l}\text { calculating the } \\
\text { quantity of } \\
\text { work }\end{array}$ & & & & & \\
\hline 22 & $\begin{array}{l}\text { Unexpected } \\
\text { weather } \\
\text { conditions } \\
\end{array}$ & 19 & 2.714 & 15 & 2.143 & 5.816 \\
\hline 23 & HSE Problems & 17 & 2.429 & 17 & 2.429 & 5.898 \\
\hline 24 & Technical issues & 18 & 2.571 & 13 & 1.857 & 4.776 \\
\hline 25 & $\begin{array}{l}\text { The occurrence } \\
\text { of differences } \\
\text { between work } \\
\text { sequences and } \\
\text { performance } \\
\text { indicators }\end{array}$ & 13 & 1.857 & 11 & 1.571 & 2.918 \\
\hline D & MAINTENANCI & & & & & \\
\hline 1 & $\begin{array}{l}\text { The quality of } \\
\text { construction is } \\
\text { ugly }\end{array}$ & 12 & 1.714 & 21 & 3 & 5.143 \\
\hline 2 & $\begin{array}{l}\text { Unexpectedly } \\
\text { severe } \\
\text { conditions }\end{array}$ & 16 & 2.286 & 17 & 2.429 & 5.551 \\
\hline 3 & $\begin{array}{l}\text { Short-term } \\
\text { focus that fails } \\
\text { for the long } \\
\text { term }\end{array}$ & 18 & 2.571 & 22 & 3.143 & 8.082 \\
\hline 4 & $\begin{array}{l}\text { Difficulties in } \\
\text { power resources }\end{array}$ & 15 & 2.143 & 15 & 2.143 & 4.592 \\
\hline 5 & $\begin{array}{l}\text { Occurrence } \\
\text { during the } \\
\text { warranty period }\end{array}$ & 16 & 2.286 & 15 & 2.143 & 4.898 \\
\hline 6 & $\begin{array}{l}\text { Traffic damage } \\
\text { occurred }\end{array}$ & 11 & 1.833 & 10 & 1.667 & 3.056 \\
\hline 7 & $\begin{array}{l}\text { Fines due to } \\
\text { response } \\
\text { Attractive less } \\
\text { quickly }\end{array}$ & 11 & 1.571 & 10 & 1.429 & 2.245 \\
\hline 8 & $\begin{array}{l}\text { Age design does } \\
\text { not fit the plan }\end{array}$ & 13 & 1.857 & 10 & 1.429 & 2.653 \\
\hline
\end{tabular}

Table. 5 Ranking of Risk

\begin{tabular}{|c|c|c|}
\hline \multicolumn{3}{|c|}{ DESIGN } \\
\hline No & Scale & Ranking \\
\hline 1 & $11-8.8$ & 1 \\
\hline 2 & $8.8-6.6$ & 2 \\
\hline 3 & $6.6-4.4$ & 3 \\
\hline 4 & $4.4-2.2$ & 4 \\
\hline 5 & $2.2-0$ & 5 \\
\hline \multicolumn{3}{|c|}{ BUILD } \\
\hline No & Scale & Ranking \\
\hline 1 & $13-10.4$ & 1 \\
\hline 2 & $10.4-7.8$ & 2 \\
\hline 3 & $7.8-5.2$ & 3 \\
\hline 4 & $5.2-2.6$ & 4 \\
\hline 5 & $2.6-0$ & 5 \\
\hline \multicolumn{3}{|c|}{ OPERATE } \\
\hline No & Scale & Ranking \\
\hline 1 & $8-6.4$ & 1 \\
\hline 2 & $6.4-4.8$ & 2 \\
\hline 3 & $4.8-3.2$ & 3 \\
\hline
\end{tabular}

\begin{tabular}{ccc}
\cline { 2 - 3 } 4 & $3.2-1.6$ & 4 \\
\hline 5 & $1.6-0$ & 5 \\
\hline \multicolumn{3}{c}{ MAINTENANCE } \\
\hline No & Scale & Ranking \\
\hline 1 & $9.00-7.20$ & 1 \\
\hline 2 & $7.20-5.40$ & 2 \\
\hline 3 & $5.40-3.60$ & 3 \\
\hline 4 & $3.60-1.80$ & 4 \\
\hline 5 & $1.80-0$ & 5 \\
\hline
\end{tabular}

Risk event results have been known variable that has the highest risk ranking with the value entered in the scale. So it is known the highest variable of each stage in Performance Based Contract.

Table. 6 The Highest Risk of each stage on Performance Based Contract

\begin{tabular}{ll}
\hline A & DESIGN \\
\hline 1 & Project budget \\
\hline 2 & Accuracy scope of work \\
\hline B & BUILD \\
\hline 1 & The vendor's offer price is higher than estimated \\
\hline C & OPERATE \\
\hline 1 & Cash flow delay \\
\hline 2 & Differences in availability of budgets with work progress \\
\hline 3 & Disturbance from the surrounding environment \\
\hline 4 & Delayed payment termin progress \\
\hline D & MAINTENANCE \\
\hline 1 & Short-term focus that fails for the long term \\
\hline
\end{tabular}

\section{CONCLUSION}

The results of the Highest Risk analysis of each stage of Performance Based Contract, such as:

1. Design :

a. Project budget (RE: 10,327)

b. Accuracy scope of work (RE: 9,388)

2. Build:

a. The vendor's offer price is higher than estimated (RE: 12.735)

3. Operate:

a. Cash flow delay (RE: 7.367)

b. Differences in availability of budgets with work progress (RE: 6.980)

c. Disturbance from the surrounding environment (RE: 6.857)

d. Delayed payment termin progress (RE: 6.592)

4. Maintenance:

a. Short-term focus that fails for the long term (RE: 8.082) 


\section{REFERENCES}

[1] YASIN, NAZARKHAN. KONTRAK KONSTRUKSI DI INDONESIA EDISI KEDUA. JAKARTA : PT GRAMEDIA PUSTAKA UTAMA, 2014.

[2] ASIYANTO. MANAJEMEN RISIKO UNTUK KONTRAKTOR. JAKARTA : PT. PRADNYA PARAMITA, 2009.

[3] Soeharto. Manajemen Proyek Jilid 1. Jakarta : Erlangga, 2001.

[4] PMI. A Guide To THE PRoJeCt MANAGEMENT BODY OF KNOWLEDGE (PMBOK GUIDE). S.L. : PROJECT MANAGEMENT InSTITUTE, 2008.

[5] Perbandingan kontrak konstruksi di Indonesia dengan kontrak konstruksi Internasional. Lestari, I Gusti Agung Ayu Istri. 2013, GaneÇ Swara, pp. 64-69.
[6] Pemodelan Hubungan Risiko Performance Based Contract Dengan Interpretive Structural Modeling (Studi Kasus Proyek Infrastruktur Jalan Di Wilayah Jawa Timur). Prihartanto, Eko and Wiguna, I Putu Artama. Surabaya: Institut Teknologi Sepuluh Nopember, 2015. Seminar Nasionas Teknik Sipil ITS XI. p. 63.

[7] Risk Management Infrastructure. Williams, T. M. 1993, International Journal of Project Management, Vol. 11, pp. 5-10. 1.

[8] Extending The Risk Process to Manage Opportunities. Hillson, D. 2002, International Journal of Project Management. 